\title{
FLAVONÓIDES EM SELEÇÕES DE ACEROLA (Malpighia sp L.). 1- TEOR DE ANTOCIANINAS E FLAVONÓIS TOTAIS
}

\author{
FLAVONOIDS IN ACEROLA (Malpighia sp L.) SELECTIONS. \\ 1- ANTHOCYANINS AND FLAVONOLS CONTENT
}

\author{
Vera Lúcia Arroxelas Galvão de Lima ${ }^{1}$ Enayde de Almeida Mélo \\ Lueci dos Santos Lima ${ }^{3}$ Poliana Pinheiro do Nascimento ${ }^{3}$
}

\begin{abstract}
Os flavonóides englobam classes de pigmentos naturais encontrados com freqüência nos vegetais. As antocianinas e os flavonóis são compostos que pertencem ao grupo dos flavonóides e são responsáveis pela coloração que varia de vermelho vivo à violeta e de branco à amarelo claro, respectivamente. A cor vermelha da acerola é decorrente da presença de antocianinas. Entretanto, a propagação da aceloreira por sementes tem gerado frutos com coloração que varia de amarelo a vermelho púrpura. Dessa forma, há o interesse em difundir plantas com produção de frutos com coloração vermelho. Este trabalho teve como objetivo determinar o teor de antocianinas e flavonóis totais nas seleções de acerola - Barbados, Coopama, Flor Branca, Inada, Miró e Okinawa. Os frutos foram colhidos nos meses de março a junho/1999, com coloração vermelha uniforme, e analisados quanto aos teores de antocianinas e flavonóis totais. As seleções Inada e Barbados apresentaram os mais altos teores desses pigmentos, quando comparados aos das demais seleções, tornando-as agronomicamente interessantes.
\end{abstract}

Palavras-chave: acerola, Malpighia sp., antocianinas, flavonóis

\section{SUMMARY}

The flavonoids comprise a class of natural pigments that are frequently found in vegetables. The anthocyanins and flavonols are compounds which belong to the flavonoids group and they are responsible for red to violet and white to lightyellow coloration, respectively. The anthocyanins pigments are responsible for the red colour of the acerola fruit. However, the seed propagation of acerola trees has grown fruits which present colour variation from yellow to dark red. So, there is interest to spread acerola trees with red colour fruits. This paper aimed to determine the content of total anthocyanins and flavonols in selections of acerola - Barbados, Coopama, Flor Branca, Inada, Miró e Okinawa. Fruits were harvested based on homogeneous red colour, between the months of March and June/1999, and analysed with regard to the content of total anthocyanins and flavonols. Inada and Barbados selections presented the highest contents of these pigments when compared to those selections what could be of agronomical interest.

Key words: west Indian cherry, Malpighia sp, anthocyanins, flavonols.

\section{INTRODUÇÃO}

A acerola (Malpighia sp L.), fruto com alto teor de vitamina $\mathrm{C}$ ( 2 a $3 \mathrm{~g} / 100 \mathrm{~g}$ de suco), vem apresentando uma rápida expansão dos plantios comerciais, tornando próspero o segmento econômico da fruticultura brasileira (OLIVEIRA \& SOARES FILHO, 1995). A acerola é matéria prima usada para a elaboração de vários produtos como polpa congelada, suco concentrado e néctar. E o consumidor espera que os produtos de acerola possuam cor próxima à do fruto in natura.

A ampla variabilidade genética, decorrente da propagação da aceroleira por semente, tem gerado frutos de coloração que varia de amarelo a vermelho púrpura. A Embrapa, visando à caracterização de germoplasma de acerola, definiu a relação de descritores mínimos para acerola no qual consta a cor vermelha como descritor da coloração da polpa do fruto maduro e, para a avaliação laboratorial, o teor total de antocianinas (OLIVEIRA et al., 1998).

\footnotetext{
${ }^{1}$ Engenheiro Químico, MSc., Professor Assistente, DCD,Universidade Federal Rural de Pernambuco (UFRPE), Av. Dom Manoel de Medeiros s/n, Dois Irmãos, 52171-900, Recife, PE. E-mail: vera @ nelore.npde.ufrpe.br. Autor para correspondência.

${ }^{2}$ Nutricionista, MSc., Profesor Adjunto, DCD, UFRPE.

${ }^{3}$ Aluno do Curso de Economia Doméstica, Bolsista PIBIC/CNPq-UFRPE. 
Os flavonóides englobam classes de pigmentos naturais encontrados com freqüência nos vegetais. As antocianinas e os flavonóis são compostos que pertencem ao grupo dos flavonóides e são responsáveis pela coloração que varia de vermelho vivo à violeta e de branco à amarelo claro, respectivamente (BOBBIO \& BOBBIO, 1995). A coloração vermelha da acerola madura é decorrente da presença de antocianinas (CHAN \& YAMAMOTO, 1994). Assim, este trabalho teve como objetivo determinar o teor de antocianinas e flavonóis totais em acerolas das seleções Barbados, Coopama, Flor Branca, Inada, Miró e Okinawa.

As acerolas foram colhidas nos meses de março, abril e junho/1999, com coloração vermelha uniforme, provenientes das seleções que irão compor o Banco de Matrizes da Universidade Federal Rural de Pernambuco (UFRPE). Os frutos foram levados ao Laboratório de Análises Físico-químicas e Sensorial de Alimentos do DCD/UFRPE e submetidos à determinação quantitativa do teor de antocianinas e flavonóis totais, utilizando o método descrito por LEES \& FRANCIS (1972), com modificações apenas no que se refere à preparação das amostras. As sementes dos frutos foram retiradas manualmente e a polpa triturada em liqüidificador. Amostras de $10 \mathrm{~g}$ de cada seleção foram homogeneizadas com $100 \mathrm{~m} \ell$ da solução extratora e estocadas durante uma noite a $4^{\circ} \mathrm{C}$.

As determinações foram efetuadas em duplicata e os resultados submetidos à análise de variância e teste de Tukey em nível 5\% de probabilidade, utilizando o programa estatístico "Minitab 10 for Windows". Foi utilizado esquema fatorial de dois fatores, um fator de 6 níveis representando as 6 seleções, e um fator de 1 nível, representando 1 estádio de maturação, perfazendo 6 tratamentos, com três repetições referentes às colheitas nos meses de março, abril e junho.

As seleções apresentaram teores de antocianinas e flavonóis totais que variaram de 14,06 a $50,98 \mathrm{mg} / 100 \mathrm{~g}$ e de 9,31 a $20,22 \mathrm{mg}$ de quercetina/100g, respectivamente (Tabela 1) Os teores de antocianinas das seleções estudadas foram maiores que os relatados por PAIVA et al. (1999) que encontraram valores de 1,97 a 46,44mg/100g. As seleções Inada e Barbados apresentaram os maiores teores de antocianinas totais $(50,98$ e $45,76 \mathrm{mg} / 100 \mathrm{~g}$, respectivamente) e estes valores estão próximos aos encontrados em frutos vermelhos como: "cranberries", 55,85mg/100g (FULEKI \& FRANCIS, 1968) e cereja ácida, 43,64mg/100g (DEKAZOS, 1970).

No que se refere aos teores de flavonóis totais, nas seleções Barbados e Inada, embora tenham apresentado os maiores teores, os valores foram estatisticamente diferentes entre si. Os teores de antocianinas e flavonóis totais das demais sele-
Tabela 1 - Teores de antocianinas e flavonóis totais em 06 seleções de acerola.

\begin{tabular}{ccc} 
Seleções & $\begin{array}{c}\text { Antocianinas totais } \\
(\mathrm{mg} / 100 \mathrm{~g})\end{array}$ & $\begin{array}{c}\text { Flavonóis totais } \\
(\mathrm{mg} \text { de quercetina/100g })\end{array}$ \\
\hline Barbados & $45,76^{\mathrm{a}}$ & $16,42^{\mathrm{b}}$ \\
Coopama & $14,06^{\mathrm{b}}$ & $10,21^{\mathrm{c}}$ \\
Flor Branca & $14,56^{\mathrm{b}}$ & $10,52^{\mathrm{c}}$ \\
Inada & $50,98^{\mathrm{a}}$ & $20,22^{\mathrm{a}}$ \\
Miró & $16,97^{\mathrm{b}}$ & $10,67^{\mathrm{c}}$ \\
Okinawa & $15,04^{\mathrm{b}}$ & $9,31^{\mathrm{c}}$ \\
\hline
\end{tabular}

As médias das amostras seguidas de letras iguais na mesma coluna não diferem entre si pelo teste de Tukey em nível de 5\% de probabilidade.

ções não apresentaram diferença significativa $(\mathrm{p}<0,05)$. A estabilidade das antocianinas ao descoramento pode ocorrer através da co-pigmentação, especialmente com os flavonóis, que exercem um ação protetora sobre as moléculas de antocianinas (BOBBIO \& BOBBIO, 1995). Dentre as seleções de acerola estudadas, a Inada e a Barbados possuem os maiores teores de antocianinas e flavonóis totais, tornando-as agronomicamente interessantes.

\section{REFERÊNCIAS BIBLIOGRÁFICAS}

BOBBIO, P.A., BOBBIO, F.O. Introdução à química de alimentos. 2.ed. São Paulo: Varela, 1995. 222p.

CHAN Jr., H.T., YAMAMOTO, H.Y. Kinetics of anthocyanin decomposition in acerola juice. ASEAN Food Journal, v.9, n.4, p.132-135, 1994.

DEKAZOS, E.D. Quantitative determination of anthocyanin pigments during the maturation and ripening of red tart cherries. Journal of Food Science. v.35, p.242-244, 1970.

FULEKI, T., FRANCIS, F.J. Quantitative methods for anthocyanins. 1. Extraction and determination of total anthocyanin in cranberries. Journal of Food Science, Chicago, v.33, p.72-77, 1968.

LEES, D.H., FRANCIS, F J. Standardization of pigment analyses in cranberries. HortScience, Alexandria, v.7, n.1, p.83-84, 1972.

OLIVEIRA, J.R.P., SOARES FILHO, W.S. Acerola: conservação, caracterização e seleção de germoplasma pelo CNPMF EMPRAPA. In: SÃO JOSÉ, A.R., ALVES, R.E. Acerola no Brasil: produção e mercado. Vitória da Conquista: DFZ/UESB, 1995. p.22-27.

OLIVEIRA, J.R.P., SOARES FILHO, W.S., NASCIMENTO, A. S., et al. Programa de pesquisa de acerola: Embrapa mandioca e fruticultura. Cruz das Almas-BA. EMBRAPA-CNPMF, 1998. 28p. (Documentos 75).

PAIVA, J.R. de, CORDEIRO, E.R., ALVES, R.E., et al. Seleção e clonagem de plantas de acerola. In: ENCONTRO DE GENÉTICA DO NORDESTE, 14, 1999, Recife, PE. Anais... Recife : Sociedade Brasileira de Genética, 1999. 87p. p.53. 